DOI: $10.17516 / 1997-1397-2021-14-3-287-300$

УДК $517.9+519.1$

\title{
A Note on the Conjugacy Between Two Critical Circle Maps
}

\author{
Utkir A. Safarov* \\ Turin Politechnic University in Tashkent \\ Tashkent, Uzbekistan \\ Tashkent State University of Economics \\ Tashkent, Uzbekistan
}

Received 10.11.2020, received in revised form 16.12.2020, accepted 04.02.2021

\begin{abstract}
We study a conjugacy between two critical circle homeomorphisms with irrational rotation number. Let $f_{i}, i=1,2$ be a $C^{3}$ circle homeomorphisms with critical point $x_{c r}^{(i)}$ of the order $2 m_{i}+1$. We prove that if $2 m_{1}+1 \neq 2 m_{2}+1$, then conjugating between $f_{1}$ and $f_{2}$ is a singular function.
\end{abstract}

Keywords: circle homeomorphism, critical point, conjugating map, rotation number, singular function.

Citation: U.A. Safarov, A Note on the Conjugacy Between Two Critical Circle Maps, J. Sib. Fed. Univ. Math. Phys., 2021, 14(3), 287-300. DOI: 10.17516/1997-1397-2021-14-3-287-300.

\section{Introduction and preliminaries}

Denjoy's classical theorem [4] states, that if the $C^{2}$ circle diffeomorphism $f$ and irrational rotation number $\rho=\rho_{f}$ then $f$ is topologically conjugate to the linear rotation $f_{\rho}$, that is, there exists a circle homeomorphism $\varphi$ with $f=\varphi^{-1} \circ f_{\rho} \circ \varphi$.

It is well known that a circle homeomorphisms $f$ with irrational rotation number is strictly ergodic, i.e. it has a unique $f$-invariant probability measure $\nu_{f}$. A remarkable fact is that the conjugacy $\varphi$ can be defined by $\varphi(x)=\nu_{f}([0, x])$, which shows, that the regularity properties of conjugacy $\varphi$ and the absolute continuity of invariant measure $\nu_{f}$ are closely related. The problem of smoothness of the conjugacy $\varphi$ for diffeomorphisms is one of the important problems of circle dynamics. The fundamental results were obtained by V. I. Arnold [1] , J. Moser [15], M. Herman [9], J. Yoccoz [17], Ya. G. Sinai and K. Khanin [12], Y. Katsnelson and D. Ornstein [13]. Notice that for sufficiently smooth circle deffeomorphisms $f$ with a typical irrational rotation number the conjugacy $\varphi$ is $C^{1}$-diffeomorphism. Consequently, the invariant measure $\nu_{f}$ is absolutely continuous with respect to Lebesgue measure $\mu$ on $S^{1}$.

Since the works of Mostow, Margulis, Sullivan, and others, rigidity problems occupy a central place in the theory of holomorphic dynamical systems. This type of problems is classical in dynamics: a rigidity theorem postulates that in a certain class of dynamical systems equivalence (combinatorial, continuous, smooth, etc.) automatically has a higher regularity. The dynamical systems considered in this paper are critical circle maps, that is smooth homeomorphisms of the circle with a single critical point having an odd type. These maps have been a subject of intensive study since the early 1980's as one of the two main examples of universality in transition to chaos. Yoccoz in [17] generalized Denjoy's classical result, a critical circle homeomorphism with irrational rotation number is topologically conjugate to an irrational rotation.

*safarovua@mail.ru

(c) Siberian Federal University. All rights reserved 
Definition 1.1. The point $x_{c r} \in S^{1}$ is called non-flat critical point of a homeomorphism $f$ with order $(2 m+1), m \in N$, if for a some $\delta$-neighborhood $U_{\delta}\left(x_{c r}\right)$, the function $f$ belongs to the class of $C^{2 m+1}\left(U_{\delta}\left(x_{c r}\right)\right)$ and

$$
f^{\prime}\left(x_{c r}\right)=f^{\prime \prime}\left(x_{c r}\right)=\cdots=f^{(2 m)}\left(x_{c r}\right)=0, \quad f^{(2 m+1)}\left(x_{c r}\right) \neq 0 .
$$

The order of the critical point $x_{c r}$ is $2 m+1$. By a critical circle map we define an orientation preserving circle homeomorphism with exactly one non-flat critical point of odd type.

An important one-parameter family of examples of critical circle maps are the Arnold's maps defined by

$$
f_{\theta}(x):=x+\theta+\frac{1}{2 \pi} \sin 2 \pi x(\bmod 1), \quad x \in S^{1} .
$$

For every $\theta \in R^{1}$ the map $f_{\theta}$ is a critical map with critical point 0 of cubic type.

Graczyk and Swiatek in [7] proved that if $f$ is $C^{3}$ smooth circle homeomorphism with finitely many critical points of polynomial type and an irrational rotation number of bounded type, then the conjugating map $\varphi$ is singular function on $S^{1}$ i.e. $\varphi^{\prime}(x)=0$ a.e. on $S^{1}$. Consequently, the invariant measure of critical circle homeomorphisms is singular w.r.t. Lebesque measure on $S^{1}$. Hence the problem of regularity of the conjugacy between two critical maps with identical irrational rotation number arises naturally. This is called the rigidity problem for critical circle homeomorphisms. For the critical circle maps the rigidity problem is developed by de Faria, de Melo, Yampolsky, Khanin and Teplinsky, Guarino among others.

The first result concerning on rigidity for critical maps was proven by de Melo and de Faria [6].

Theorem 1.1 (see [6]). If $f_{1}, f_{2}$ are $C^{3}$ critical circle mappings with the same irrational rotation number of bounded type and the same power-law at the critical point, then there exists a $C^{1+\alpha}$ conjugacy $h$ between $f_{1}$ and $f_{2}$ for some universal $\alpha>0$.

The following result of D. Khmelev and M. Yampolski [14] seemed to indicate that the analytic case could be different.

Theorem 1.2 ([14]). There exists a universal constant $\alpha>0$ such that the following holds. Let $f_{1}$ and $f_{2}$ be two analytic critical circle maps with the same irrational rotation number. Denote $h: S^{1} \rightarrow S^{1}$ conjugacies between $f_{1}$ and $f_{2}$ fixing the critical points. Then $h$ is $C^{1+\alpha}$ at the critical point.

K. Khanin and A. Teplinskii [11] proved that any two $f_{1}$ and $f_{2}$ analytic critical circle maps with the same order of critical points and the same irrational rotation number are $C^{1}$-smoothly conjugate to each other. Later, A. Avila [2] showed, that there exist $f_{1}$ and $f_{2}$ analytic homeomorphisms with the same irrational rotation number such that $h$ is not $C^{1+\alpha}$ for any $\alpha>0$.

Next we formulate the result of P. Guarino, M. Martens, and W. de Melo [8].

Theorem $1.3([8])$. Let $f_{1}$ and $f_{2}$ be two analytic $C^{4}$-circle homeomorphisms with the same irrational rotation number and with a unique critical point of the same odd type. Then they are $C^{1}$-smoothly conjugate to each other. The conjugacy is $C^{1+\alpha}$ for Lebesgue almost every rotation number.

The present work continuous and completes the above results. Namely we show that if the rotation numbers of two critical homeomorphisms coincide but the orders of critical points are different then the conjugacy $h$ is a singular function. Now we formulate our main result.

Theorem 1.4. Let $f_{1}$ and $f_{2}$ be $C^{3}$ critical circle maps with the same irrational rotation number. Suppose that the orders of critical points of $f_{1}$ and $f_{2}$ are different i.e. $2 m_{1}+1 \neq 2 m_{2}+1$. Then the conjugacy $h$ between $f_{1}$ and $f_{2}$ is a singular function on $S^{1}$. 


\section{Notations, terminalogy, background}

Let $f$ be a circle homeomorphism that preserves orientation, i.e. $f(x)=F(x)(\bmod 1), x \in$ $S^{1} \simeq[0,1)$, where $F$ is continuous, strictly increasing on $R^{1}$ and $F(x+1)=F(x)+1$ for any $x \in R$. $F$ is called lift of homeomorphism $f$. The important characteristic of the circle homeomorphism $f$ is it's rotation number (see for instance [6]) $\rho_{f}$ which defined by $\rho_{f}=\lim _{n \rightarrow \infty} \frac{F^{n}(x)}{n}(\bmod 1)$, here and later $F^{n}$ denotes the $n$-th iteration of $F$. The rotation number $\rho_{f}$ is rational if and only if $f$ has periodic orbits.

2.1. Dynamical partition. Let $f$ be an orientation preserving homeomorphism of the circle with lift $F$ and irrational rotation number $\rho=\rho_{f}$. We denote by $\left\{a_{n}, n \in \mathbb{N}\right\}$ the sequence of entries in the continued fraction expansion of $\rho$, i.e. $\rho=\left[a_{1}, a_{2}, \ldots, a_{n}, \ldots\right]$. Denote by $p_{n} / q_{n}=\left[a_{1}, a_{2}, \ldots, a_{n}\right]$ the convergents of $\rho$. Their denominators $q_{n}$ satisfy the recurrence relation, that is $q_{n+1}=a_{n+1} q_{n}+q_{n-1}, n \geqslant 1, q_{0}=1, q_{1}=a_{1}$.

For an arbitrary point $x_{0} \in S^{1}$ we define $\Delta_{0}^{(n)}\left(x_{0}\right)$ the closed interval on $S^{1}$ with endpoints $x_{0}$ and $x_{q_{n}}=f^{q_{n}}\left(x_{0}\right)$. Note that for odd $n$ the point $x_{q_{n}}$ lies to the left of $x_{0}$ and for even $n$ to the right. Denote by $\Delta_{i}^{(n)}\left(x_{0}\right)$ the iterates of the interval $\Delta_{0}^{(n)}\left(x_{0}\right)$ under $f: \Delta_{i}^{(n)}\left(x_{0}\right):=$ $f^{i}\left(\Delta_{0}^{(n)}\left(x_{0}\right)\right), i \geqslant 1$.

Lemma 2.1 (see [12]). Consider an arbitrary point $x_{0} \in S^{1}$. A finite piece $\left\{x_{i}, 0 \leqslant i<\right.$ $\left.q_{n}+q_{n-1}\right\}$ of the trajectory of this point divides the circle into the following disjoint (except for the endpoints) intervals: $\Delta_{i}^{(n-1)}\left(x_{0}\right), 0 \leqslant i<q_{n}, \Delta_{j}^{(n)}\left(x_{0}\right), 0 \leqslant j<q_{n-1}$.

We denote the obtained partition by $\xi_{n}\left(x_{0}\right)$ and call it $n$-th dynamical partition of the circle. We now briefly describe the process of transition from $\xi_{n}\left(x_{0}\right)$ to $\xi_{n+1}\left(x_{0}\right)$. All intervals $\Delta_{j}^{(n)}\left(x_{0}\right), 0 \leqslant j<q_{n-1}$, are preserved, and each of the intervals $\Delta_{i}^{(n-1)}\left(x_{0}\right)$ is divided into $a_{n+1}+1$ sub intervals:

$$
\Delta_{i}^{(n-1)}\left(x_{0}\right)=\Delta_{i}^{(n+1)}\left(x_{0}\right) \cup \bigcup_{s=0}^{a_{n+1}-1} \Delta_{i+q_{n-1}+s q_{n}}^{(n)}\left(x_{0}\right) .
$$

Obviously one has $\xi_{1}\left(x_{0}\right) \leqslant \xi_{2}\left(x_{0}\right) \leqslant \ldots \leqslant \xi_{n}\left(x_{0}\right) \leqslant \ldots$

Definition 2.1. Let $K>1$ be a constant. We call two intervals $I_{1}$ and $I_{2}$ of $S^{1}$ are $K$ comparable, if the inequalities $K^{-1} \mu\left(I_{2}\right) \leqslant \mu\left(I_{1}\right) \leqslant K \mu\left(I_{2}\right)$ hold.

Next we formulate the lemma, that is proved in the similar way as in [16].

Let $x_{c r} \in S^{1}$ be a critical point of homeomorphism $f$. For any $x_{0} \in S^{1}$, consider the dynamical partition $\xi_{n}\left(x_{0}\right)$. For definiteness we assume that $n$ is odd. Then $x_{q_{n}} \prec x_{0} \prec x_{q_{n-1}}$. The structure of the dynamical partition implies that $\bar{x}_{c r}=f^{-p}\left(x_{c r}\right) \in\left[x_{q_{n}}, x_{q_{n-1}}\right]$, for some $p, 0<p<q_{n}$. Let $I_{1}$ and $I_{2}$ be any elements of a dynamical partition $\xi_{m}\left(\bar{x}_{c r}\right), m \geqslant n$ having a common endpoints.

Lemma 2.2. Let $f \in C^{3}\left(S^{1}\right)$ be a critical circle homeomorphism with irrational rotation number. Then there exists a constant $K>1$ depending only on $f$ such that the intervals $I_{1}$ and $I_{2}$ are $K$-comparable.

It follows from the Lemma 2.2 that the trajectory of each point is dense in $S^{1}$. Hence it follows that there exists conjugation map $\varphi$ between $f$ and $f_{\rho}$, i.e. $\varphi(f(x))=f_{\rho}(\varphi(x))$ for any $x \in S^{1}$. 
We assume that $\Delta^{(m+k)}$ is element of partitioning $\xi_{m+k}\left(\bar{x}_{c r}\right)$, while $\Delta^{(m)}$ is an element of partitioning $\xi_{m}\left(\bar{x}_{c r}\right)$ that contains $\Delta^{(m+k)}$.

Lemma 2.3 (see [10]). There exist constants $\lambda_{1}(f)<\lambda_{2}(f)<1$ such that

$$
\ell\left(\Delta^{(m+k)}\right) \leqslant \text { const } \lambda_{2}^{k}(f) \ell\left(\Delta^{(m)}\right), \quad \ell\left(\Delta_{0}^{(m)}\right) \geqslant \text { const } \lambda_{1}^{m}(f) .
$$

2.2. Cross-ratio tools. In the proof of our main theorem the tool of cross-ratio plays a key role.

Definition 2.2. The cross-ratio of four points $\left(z_{1}, z_{2}, z_{3}, z_{4}\right), z_{1}<z_{2}<z_{3}<z_{4}$ is the number

$$
\operatorname{Cr}\left(z_{1}, z_{2}, z_{3}, z_{4}\right)=\frac{\left(z_{2}-z_{1}\right)\left(z_{4}-z_{3}\right)}{\left(z_{3}-z_{1}\right)\left(z_{4}-z_{2}\right)}
$$

Definition 2.3. Given four real numbers $\left(z_{1}, z_{2}, z_{3}, z_{4}\right)$ with $z_{1}<z_{2}<z_{3}<z_{4}$ and a strictly increasing function $F: \mathbb{R}^{1} \rightarrow \mathbb{R}^{1}$. The distortion of their cross-ratio under $F$ is given by

$$
\operatorname{Dist}\left(z_{1}, z_{2}, z_{3}, z_{4} ; F\right)=\frac{C r\left(F\left(z_{1}\right), F\left(z_{2}\right), F\left(z_{3}\right), F\left(z_{4}\right)\right)}{C r\left(z_{1}, z_{2}, z_{3}, z_{4}\right)} .
$$

For $m \geqslant 3$ and $z_{i} \in S^{1}, 1 \leqslant i \leqslant m$, suppose that $z_{1} \prec z_{2} \prec \cdots \prec z_{m} \prec z_{1}$ (in the sense of the ordering on the circle). Then we set $\hat{z}_{1}:=z_{1}$ and

$$
\hat{z}_{i}:= \begin{cases}z_{i} & \text { if } z_{1}<z_{i}<1 \\ 1+z_{i} & \text { if } 0<z_{i}<z_{1}\end{cases}
$$

for $2 \leqslant i \leqslant m$.

Obviously, $\hat{z}_{1}<\hat{z}_{2}<\ldots<\hat{z}_{m}$. The vector $\left(\hat{z}_{1}, \hat{z}_{2}, \ldots, \hat{z}_{m}\right)$ is called the lifted vector of $\left(z_{1}, z_{2}, \ldots, z_{m}\right) \in\left(S^{1}\right)^{m}$.

Let $f$ be a circle homeomorphism with lift $F$. We define the cross-ratio distortion of $\left(z_{1}, z_{2}, z_{3}, z_{4}\right), z_{1} \prec z_{2} \prec z_{3} \prec z_{4} \prec z_{1}$ with respect to $f$ by $\operatorname{Dist}\left(z_{1}, z_{2}, z_{3}, z_{4} ; f\right)=$ $=\operatorname{Dist}\left(\hat{z}_{1}, \hat{z}_{2}, \hat{z}_{3}, \hat{z}_{4} ; F\right)$, where $\left(\hat{z}_{1}, \hat{z}_{2}, \hat{z}_{3}, \hat{z}_{4}\right)$ is the lifted vector of $\left(z_{1}, z_{2}, z_{3}, z_{4}\right)$. We need the following lemma.

Lemma $2.4([5])$. Let $z_{i} \in S^{1}, i=1,2,3,4, z_{1} \prec z_{2} \prec z_{3} \prec z_{4}$. Consider a circle homeomorphism $f$ with $f \in C^{2+\varepsilon}\left(\left[z_{1}, z_{4}\right]\right), \varepsilon>0$, and $f^{\prime}(x) \geqslant$ const $>0$ for $x \in\left[z_{1}, z_{4}\right]$. Then there is a positive constant $C_{1}=C_{1}(f)$ such that

$$
\left|\operatorname{Dist}\left(z_{1}, z_{2}, z_{3}, z_{4} ; f\right)-1\right| \leqslant C_{1}\left|\hat{z}_{4}-\hat{z}_{1}\right|^{1+\varepsilon}
$$

where $\left(\hat{z}_{1}, \hat{z}_{2}, \hat{z}_{3}, \hat{z}_{4}\right)$ is the lifted vector of $\left(z_{1}, z_{2}, z_{3}, z_{4}\right)$.

We now consider the case when the interval $\left[z_{1}, z_{4}\right]$ contains a critical point $x_{c r}$ of the homeomorphism $f$. More precisely, suppose that $z_{2}=x_{c r}$. We define numbers $\alpha, \beta, \gamma, \xi$ and $\eta$ as follows:

$$
\alpha:=\hat{z}_{2}-\hat{z}_{1}, \quad \beta:=\hat{z}_{3}-\hat{z}_{2}, \quad \gamma:=\hat{z}_{4}-\hat{z}_{3}, \quad \xi:=\frac{\beta}{\alpha}, \quad \eta:=\frac{\beta}{\gamma},
$$

where $\left(\hat{z}_{1}, \hat{z}_{2}, \hat{z}_{3}, \hat{z}_{4}\right)$ is the lifted vector of $\left(z_{1}, z_{2}, z_{3}, z_{4}\right)$.

Thus we need the following lemma. 
Lemma 2.5. Suppose that a homeomorphism $f$ with lift $F$ has a critical point $x_{c r}$ with order $2 m+1, m \in N$. Then for any $\varepsilon>0$, there exist $\delta=\delta(\varepsilon)>0$, such that for all $z_{i} \in U_{\delta}\left(x_{c r}\right)$, $i=\overline{1, n}, z_{1} \prec z_{2}=x_{c r} \prec z_{3} \prec z_{4}$ one has

$$
\left|\operatorname{Dist}\left(z_{1}, z_{2}, z_{3}, z_{4} ; f\right)-\frac{1}{1-\xi+\xi^{2}-\cdots+\xi^{2 m}} \times \frac{e_{2 m} \eta^{2 m}+e_{2 m-1} \eta^{2 m-1}+\cdots+e_{1} \eta+1}{\eta^{2 m}+C_{2 m}^{1} \eta^{2 m-1}+\cdots+C_{2 m}^{2 m-1} \eta+1}\right|<R_{0} \varepsilon,
$$

where the constants $e_{2 m}=2 m+1, e_{i}=C_{2 m}^{i}+C_{2 m-1}^{i-1}+\cdots+C_{2 m-i}^{0}$ and $R_{0}$ depends only on function $f$.

Proof. Fix a number $\varepsilon$. It is easy to check that for any $z_{i} \in S^{1}, i=\overline{1, n}, z_{1} \prec z_{2} \prec z_{3} \prec z_{4}$ one has

$$
\begin{aligned}
& F\left(z_{1}\right)=F\left(\hat{z}_{2}\right)-F^{\prime}\left(\hat{z}_{2}\right)\left(\hat{z}_{2}-\hat{z}_{1}\right)+\cdots+\frac{F^{(2 m)}\left(\hat{z}_{2}\right)}{2 m !}\left(\hat{z}_{2}-\hat{z}_{1}\right)^{2 m}-\frac{1}{2 m !} \int_{\hat{z}_{1}}^{\hat{z}_{2}} F^{(2 m+1)}(y)\left(y-\hat{z}_{1}\right)^{2 m} d y, \\
& F\left(\hat{z}_{s}\right)=F\left(\hat{z}_{2}\right)+F^{\prime}\left(\hat{z}_{2}\right)\left(\hat{z}_{s}-\hat{z}_{2}\right)+\cdots+\frac{F^{(2 m)}\left(\hat{z}_{2}\right)}{2 m !}\left(\hat{z}_{s}-\hat{z}_{2}\right)^{2 m}+ \\
& +\frac{1}{2 m !} \int_{\hat{z}_{2}}^{\hat{z}_{s}} F^{(2 m+1)}(y)\left(\hat{z}_{s}-y\right)^{2 m} d y, \quad s=3,4 .
\end{aligned}
$$

By the assumption of the lemma, $z_{2}=x_{c r}$, and using the (2.1) we write $\operatorname{Cr}\left(f\left(z_{1}\right), f\left(z_{2}\right), f\left(z_{3}\right), f\left(z_{4}\right)\right)$ as follows

$$
\begin{gathered}
C r\left(f\left(z_{1}\right), f\left(z_{2}\right), f\left(z_{3}\right), f\left(z_{4}\right)\right)=\frac{\left(F\left(\hat{z}_{2}\right)-F\left(\hat{z}_{1}\right)\right)\left(F\left(\hat{z}_{4}\right)-F\left(\hat{z}_{3}\right)\right)}{\left(F\left(\hat{z}_{3}\right)-F\left(\hat{z}_{1}\right)\right)\left(F\left(\hat{z}_{4}\right)-F\left(\hat{z}_{2}\right)\right)}= \\
=\frac{\int_{\hat{z}_{1}}^{\hat{z}_{2}} F^{(2 m+1)}(y)\left(y-\hat{z}_{1}\right)^{2 m} d y}{\int_{\hat{z}_{2}}^{\hat{z}_{3}} F^{(2 m+1)}(y)\left(\hat{z}_{3}-y\right)^{2 m} d y+\int_{\hat{z}_{1}}^{\hat{z}_{2}} F^{(2 m+1)}(y)\left(y-\hat{z}_{1}\right)^{2 m} d y} \times \\
\times \frac{\int_{\hat{z}_{2}}^{\hat{z}_{4}} F^{(2 m+1)}(y)\left(\hat{z}_{4}-y\right)^{2 m} d y-\int_{\hat{z}_{2}}^{\hat{z}_{3}} F^{(2 m+1)}(y)\left(\hat{z}_{3}-y\right)^{2 m} d y}{\int_{\hat{z}_{2}}^{\hat{z}_{4}} F^{(2 m+1)}(y)\left(\hat{z}_{4}-y\right)^{2 m} d y},
\end{gathered}
$$

where $\left(\hat{z}_{1}, \hat{z}_{2}, \hat{z}_{3}, \hat{z}_{4}\right)$ is the lifted vector of $\left(z_{1}, z_{2}, z_{3}, z_{4}\right)$. Since $F^{(2 l+1)} \in C\left(U_{\omega}\left(x_{c r}\right)\right)$, there exist $\delta(\varepsilon)>0$, such that for any $x, y \in\left(x_{c r}-\omega, x_{c r}+\omega\right)$ the inequality $\left|F^{(2 m+1)}(x)-F^{(2 m+1)}(y)\right|<\varepsilon$ is true.

Hence from (2.2) we have

$$
\begin{gathered}
\operatorname{Cr}\left(f\left(z_{1}\right), f\left(z_{2}\right), f\left(z_{3}\right), f\left(z_{4}\right)\right)= \\
=\frac{\int_{\hat{z}_{1}}^{\hat{z}_{2}} F^{(2 m+1)}\left(x_{c r}\right)\left(y-\hat{z}_{1}\right)^{2 m} d y(1+O(\varepsilon))}{\left(\int_{\hat{z}_{2}}^{\hat{z}_{3}} F^{(2 m+1)}\left(x_{c r}\right)\left(\hat{z}_{3}-y\right)^{2 m} d y+\int_{\hat{z}_{1}}^{\hat{z}_{2}} F^{(2 m+1)}\left(x_{c r}\right)\left(y-\hat{z}_{1}\right)^{2 m} d y\right)(1+O(\varepsilon))} \times \\
\times \frac{\left(\int_{\hat{z}_{2}}^{\hat{z}_{4}} F^{(2 m+1)}\left(x_{c r}\right)\left(\hat{z}_{4}-y\right)^{2 m} d y-\int_{\hat{z}_{2}}^{\hat{z}_{3}} F^{(2 m+1)}\left(x_{c r}\right)\left(\hat{z}_{3}-y\right)^{2 m} d y\right)(1+O(\varepsilon))}{\int_{\hat{z}_{2}} F^{(2 m+1)}\left(x_{c r}\right)\left(\hat{z}_{4}-y\right)^{2 m} d y(1+O(\varepsilon))}=
\end{gathered}
$$




$$
=\frac{\alpha^{2 m+1}}{\alpha^{2 m+1}+\beta^{2 m+1}} \cdot \frac{(\gamma+\beta)^{2 m+1}-\beta^{2 m+1}}{(\gamma+\beta)^{2 m+1}}(1+O(\varepsilon)) .
$$

From the last equality it follows that

$$
\begin{gathered}
\operatorname{Dist}\left(z_{1}, z_{2}, z_{3}, z_{4} ; f\right)=\frac{1}{1-\xi+\xi^{2}-\cdots+\xi^{2 m}} \times \\
\times \frac{(1+\eta)^{2 m}+(1+\eta)^{2 m-1} \eta+\cdots+(1+\eta) \eta^{2 m-1}+\eta^{2 m}}{(1+\eta)^{2 m}}(1+O(\varepsilon))= \\
=\frac{1}{1-\xi+\xi^{2}-\cdots+\xi^{2 m}} \times \frac{e_{2 m} \eta^{2 m}+e_{2 m-1} \eta^{2 m-1}+\cdots+e_{1} \eta+1}{\eta^{2 m}+C_{2 m}^{1} \eta^{2 m-1}+\cdots+C_{2 m}^{2 m-1} \eta+1}(1+O(\varepsilon)) .
\end{gathered}
$$

Thus Lemma 2.5 is proved.

Next suppose the interval $\left[z_{1}, z_{4}\right]$ is a subset of the interval $U_{\omega}\left(x_{c r}\right)$ but does not contain a critical point $x_{c r}$ of the homeomorphism $f$. Let $d=\min _{1 \leqslant s \leqslant 4} \ell\left(\left[z_{s}, x_{c r}\right]\right)$. We now state an assertion from [10].

Lemma 2.6 (see [10]). Suppose that a homeomorphism $f$ satisfies the conditions of Lemma 2.5. Then the following equality holds

$$
\operatorname{Dist}\left(z_{1}, z_{2}, z_{3}, z_{4} ; f\right)=1+O\left(\left(\frac{\alpha+\beta+\gamma}{d}\right)^{2}\right) .
$$

\section{Proof of Theorem $\mathbf{1 . 4}$}

In order to prove Theorem 1.4 we need several lemmas which we formulate next. Their proofs will be given later. We consider two copies of the unit circle $S^{1}$. The homeomorphism $f_{1}$ acts on the first circle and $f_{2}$ acts on the second one. Assume that $f_{i}, i=1,2$ satisfies the conditions of Theorem 1.4.

Let $\varphi_{1}$ and $\varphi_{2}$ be conjugations of $f_{1}$ and $f_{2}$ to linear rotation $f_{\rho}$, i.e. $\varphi_{1} \circ f_{1}=f_{\rho} \circ \varphi_{1}$ and $\varphi_{2} \circ f_{2}=f_{\rho} \circ \varphi_{2}$. It is easy to check that the homeomorphisms $f_{1}$ and $f_{2}$ are conjugated by $h=\varphi_{2} \circ \varphi_{1}^{-1}$, i. e. $h \circ f_{1}(x)=f_{2} \circ h(x), \forall x \in S^{1}$. Recall that every $\varphi_{i}, i=1,2$ is unique up to an additional constant. This gives us a possibility to choose $h$ with initial condition $h\left(x_{c r}^{(1)}\right)=x_{c r}^{(2)}$.

Notice the conjugation $h(x)$ is continuous function on $S^{1}$. It suffices to show that $h^{\prime}(x)=0$ for almost all $x$ with respect to the Lebesgue measure. The derivative $h^{\prime}(x)=0$ exists for almost all $x$ with respect to the Lebesgue measure because the function $h$ is monotonic. Let us show that $h^{\prime}(x)=0$ at all points where the derivative is defined.

Lemma 3.1 (see [5]). Assume, that the conjugating homeomorphism $h(x)$ has a positive derivative $h^{\prime}\left(x_{0}\right)=\omega_{0}$ at some point $x_{0} \in S^{1}$, and that the following conditions hold for the points $z_{i} \in S^{1}, i=1, \ldots, 4$, with $z_{1} \prec z_{2} \prec z_{3} \prec z_{4}$, and some constant $R_{1}>1$ :

(a) the intervals $\left[z_{1}, z_{2}\right],\left[z_{2}, z_{3}\right],\left[z_{3}, z_{4}\right]$ are pairwise $R_{1}$-comparable;

(b) $\max _{1 \leqslant i \leqslant 4} \ell\left(\left[z_{i}, x_{0}\right]\right) \leqslant R_{1} \ell\left(\left[z_{1}, z_{2}\right]\right)$.

Then for any $\varepsilon>0$ there exists $\delta=\delta(\varepsilon)>0$ such that

$$
\left|\operatorname{Dist}\left(z_{1}, z_{2}, z_{3}, z_{4} ; h\right)-1\right| \leqslant C_{2} \varepsilon,
$$

if $z_{i} \in\left(x_{0}-\delta, x_{0}+\delta\right)$ for all $i=1,2,3,4$, where the constant $C_{2}>0$ depends only on $R_{1}, \omega_{0}$ and not on $\varepsilon$. 
Suppose that $h^{\prime}\left(x_{0}\right)=\omega_{0}$, where $x_{0} \in S^{1}$. Let $\xi_{n}\left(x_{0}\right)$ be its $n$-th dynamical partition. Put $t_{0}:=h\left(x_{0}\right)$ and consider the dynamical partition $\tau_{n}\left(t_{0}\right)$ of $t_{0}$ on the second circle determined by the homeomorphism $f_{2}$, i.e.

$$
\tau_{n}\left(t_{0}\right)=\left\{I_{i}^{(n-1)}\left(t_{0}\right), \quad 0 \leqslant i \leqslant q_{n}-1\right\} \cup\left\{I_{j}^{(n)}\left(t_{0}\right), \quad 0 \leqslant j \leqslant q_{n-1}-1\right\}
$$

with $I_{0}^{(n)}\left(t_{0}\right)$ the closed interval with endpoints $t_{0}$ and $f_{2}^{q_{n}}\left(t_{0}\right)$. Choose an odd natural number $n_{1}=n\left(f_{1}, f_{2}\right)$ such that the $n_{1}$-th renormalization neighborhoods $\left[x_{q_{n_{1}}}, x_{q_{n_{1}-1}}\right]$ and $\left[t_{q_{n_{1}}}, t_{q_{n_{1}-1}}\right]$ do not contain critical point of $f_{1}$ and $f_{2}$ respectively. Since the identical rotation number $\rho$ of $f_{1}$ and $f_{2}$ is irrational, the order of the points on the orbit $\left\{f_{1}^{k}\left(x_{0}\right), k \in \mathbb{Z}\right\}$ on the first circle will be precisely the same as the one for the orbit $\left\{f_{2}^{k}\left(t_{0}\right), k \in \mathbb{Z}\right\}$ on the second one. This together with the relation $h\left(f_{1}(x)\right)=f_{2}(h(x))$ for $x \in S^{1}$ implies that

$$
h\left(\Delta_{i}^{\left(n_{1}-1\right)}\right)=I_{i}^{\left(n_{1}-1\right)}, \quad 0 \leqslant i \leqslant q_{n_{1}}-1, \quad h\left(\Delta_{j}^{\left(n_{1}\right)}\right)=I_{j}^{\left(n_{1}\right)}, \quad 0 \leqslant j \leqslant q_{n_{1}-1}-1 .
$$

The structure of the dynamical partitions implies that $\bar{x}_{c r}^{(1)}\left(n_{1}\right)=f_{1}^{-l}\left(x_{c r}^{(1)}\right) \in\left[x_{q_{n_{1}}}, x_{q_{n_{1}-1}}\right]$, where $l \in\left(0, q_{n_{1}-1}\right)$ if $\bar{x}_{c r}^{(1)}\left(n_{1}\right) \in\left[x_{q_{n_{1}}}, x_{0}\right]$, and $l \in\left(0, q_{n_{1}}\right)$ if $\bar{x}_{c r}^{(1)}\left(n_{1}\right) \in\left[x_{0}, x_{q_{n_{1}-1}}\right]$. Since $h$ conjugation between $f_{1}$ and $f_{2}$, we get

$$
f_{2}^{l}\left(h\left(\bar{x}_{c r}^{(1)}\right)\right)=f_{2}^{l-1}\left(f_{2}\left(h\left(\bar{x}_{c r}^{(1)}\right)\right)\right)=f_{2}^{l-1}\left(h\left(f_{1}\left(\bar{x}_{c r}^{(1)}\right)\right)\right)=\cdots=h\left(f_{1}^{l}\left(\bar{x}_{c r}^{(1)}\right)\right)=h\left(x_{c r}^{(1)}\right)=x_{c r}^{(2)} .
$$

Hence $\bar{x}_{c r}^{(2)}\left(n_{1}\right)=f_{2}^{-l}\left(x_{c r}^{(2)}\right) \in\left[t_{q_{n_{1}}}, t_{q_{n_{1}-1}}\right]$. The points $\bar{x}_{c r}^{(1)}\left(n_{1}\right)$ and $\bar{x}_{c r}^{(2)}\left(n_{1}\right)$ are called the $q_{n_{1}}$-pre-images of the critical points $x_{c r}^{(1)}$ and $x_{c r}^{(2)}$, respectively.

Next we introduce the concept of a "regular" cover of the critical point. Let $z_{i} \in S^{1}, i=\overline{1,4}$, $z_{1} \prec z_{2} \prec z_{3} \prec z_{4} \prec z_{1}$. Define for each $j, 0<j<q_{n}$

$$
\xi_{f_{1}}(j)=\frac{\ell\left(\left[f_{1}^{j}\left(z_{2}\right), f_{1}^{j}\left(z_{3}\right)\right]\right)}{\ell\left(\left[f_{1}^{j}\left(z_{1}\right), f_{1}^{j}\left(z_{2}\right)\right]\right)}, \quad \eta_{f_{1}}(j)=\frac{\ell\left(\left[f_{1}^{j}\left(z_{2}\right), f_{1}^{j}\left(z_{3}\right)\right]\right)}{\ell\left(\left[f_{1}^{j}\left(z_{3}\right), f_{1}^{j}\left(z_{4}\right)\right]\right)} .
$$

Definition 3.1. Let $M>1, \zeta \in(0,1), \delta>0$ be constant numbers, $n$ is a positive integer and $x_{0} \in S^{1}$. We say that a triple of intervals $\left(\left[z_{1}, z_{2}\right],\left[z_{2}, z_{3}\right],\left[z_{3}, z_{4}\right]\right), z_{i} \in S^{1}, i=1,2,3,4$, covers the critical point of $x_{c r}^{(1)} "\left(M, \zeta, \theta, \delta ; x_{0}\right)$-regularly", if the following conditions hold:

1) $\left[z_{1}, z_{4}\right] \subset\left(x_{0}-\delta, x_{0}+\delta\right)$, and the system of intervals $\left\{f_{1}^{j}\left(\left[z_{1}, z_{4}\right]\right), 0 \leqslant j \leqslant q_{n}-1\right\}$ cover critical point $x_{c r}^{(1)}$ only once;

2) $z_{2}=f_{1}^{-l}\left(x_{c r}^{(1)}\right)$ for some $l, 0<l<q_{n}$;

3) $\xi_{f_{1}}(l)<\zeta$ and $\eta_{f_{1}}(l) \geqslant M$.

Denote

$$
L=\min \left\{2 m_{1}+1,2 m_{2}+1,2\left|m_{1}-m_{2}\right|\right\} .
$$

Lemma 3.2. Suppose that the homeomorphisms $f_{i}, i=1,2$ satisfy the conditions of Theorem 1.4. Then for any $x_{0} \in S^{1}$ and $\delta>0$ there exist constant $M_{0}>1$ and $\zeta_{0} \in(0,1)$, such that for all triples of intervals $\left[z_{s}, z_{s+1}\right] \subset\left(x_{0}-\delta, x_{0}+\delta\right), s=1,2,3$, and $\left[h\left(z_{s}\right), h\left(z_{s+1}\right)\right], s=1,2,3$, covering the critical points $x_{c r}^{(1)}$ and $x_{c r}^{(2)}$ regularly with constants $M_{0}$ and $\zeta_{0}$ the following inequalities hold:

$$
\left|\frac{1}{1-\xi_{f_{1}}(l)+\cdots+\xi_{f_{1}}^{2 m_{1}}(l)} \times \frac{e_{2 m_{1}} \eta_{f_{1}}^{2 m_{1}}(l)+e_{2 m_{1}-1} \eta_{f_{1}}^{2 m_{1}-1}(l)+\cdots+1}{\eta_{f_{1}}^{2 m_{1}}(l)+C_{2 m_{1}}^{1} \eta_{f_{1}}^{2 m_{1}-1}(l)+\cdots+1}-\left(2 m_{1}+1\right)\right|<\frac{L}{16},
$$




$$
\left|\frac{1}{1-\xi_{f_{2}}(l)+\cdots+\xi_{f_{2}}^{2 m_{2}}(l)} \times \frac{e_{2 m_{2}} \eta_{f_{2}}^{2 m_{2}}(l)+e_{2 m_{2}-1} \eta_{f_{2}}^{2 m_{2}-1}(l)+\cdots+1}{\eta_{f_{2}}^{2 m_{2}}(l)+C_{2 m_{2}}^{1} \eta_{f_{2}}^{2 m_{2}-1}(l)+\cdots+1}-\left(2 m_{2}+1\right)\right|<\frac{L}{16},
$$

where $m_{1}$ and $m_{2}$ are orders of critical points $x_{c r}^{(1)}$ and $x_{c r}^{(2)}$ respectively.

Assume that the homeomorphism $f_{1}$ satisfies the conditions of Theorem 1.4. Let $\xi_{n}\left(x_{c r}^{(1)}\right)$ be a dynamical partition of the circle by $f_{1}$. We take a natural number $r$, such that $\Delta_{0}^{(r)}\left(x_{c r}^{(1)}\right) \cup$ $\Delta_{0}^{(r-1)}\left(x_{c r}^{(1)}\right) \subset U_{\omega_{1}}\left(x_{c r}^{(1)}\right)$. Suppose that $h^{\prime}\left(x_{0}\right)=p_{0}>0$ for some $x_{0} \in S^{1}$. Consider the dynamical partition $\xi_{n}\left(x_{0}\right)$ of the point $x_{0}$ under $f_{1}$. Suppose that $n>r$ an odd natural number. Let $\bar{x}_{c r}^{(1)}=f^{-l}\left(x_{c r}^{(1)}\right) \in\left[x_{q_{n}}, x_{q_{n-1}}\right]$.

Let $\left\{\xi_{n+k}\left(\bar{x}_{c r}^{(1)}\right)\right\}_{k=0}^{\infty}$ be a sequence of dynamical partitions of the point $\bar{x}_{c r}$. We define the points $z_{i}, i=1,2,3,4$ as follows

$$
z_{1}=f^{q_{n+k_{0}}}\left(\bar{x}_{c r}^{(1)}\right), \quad z_{2}=\bar{x}_{c r}^{(1)}, \quad z_{3}=f^{q_{n+k_{0}+k_{1}}}\left(\bar{x}_{c r}^{(1)}\right), \quad z_{4}=f^{q_{n+k_{0}+k_{1}}+q_{n+k_{2}}}\left(\bar{x}_{c r}^{(1)}\right) .
$$

Lemma 3.3. Suppose that the homeomorphisms $f_{1}$ and $f_{2}$ satisfies the conditions of Theorem 1.4. Let $h^{\prime}\left(x_{0}\right)=p_{0}>0$ for some $x_{0} \in S^{1}, \delta \in(0,1)$ and $k_{0} \in N$. Then there exist natural numbers $k_{1}, k_{2}$ such that for sufficiently large $n$, the triple of intervals $\left[z_{s}, z_{s+1}\right] \subset\left(x_{0}-\delta, x_{0}+\delta\right)$, $s=1,2,3$ satisfies the following properties:

(1) the intervals $\left\{\left[f_{1}^{j}\left(z_{1}\right), f_{1}^{j}\left(z_{4}\right)\right], 0 \leqslant j \leqslant q_{n}\right\}$ cover each point at most once;

(2) the intervals $\left[z_{s}, z_{s+1}\right]$ and $\left[f_{1}^{q_{n}}\left(z_{s}\right), f_{1}^{q_{n}}\left(z_{s+1}\right)\right], s=1,2,3$ satisfy conditions (a) and (b) of Lemma 3.1 with some constant $R_{1}>1$ depending on $k_{0}, k_{1}, k_{2}$;

(3) the triples of intervals $\left(\left[z_{s}, z_{s+1}\right], s=1,2,3\right)$ and $\left(\left[h\left(z_{s}\right), h\left(z_{s+1}\right)\right], s=1,2,3\right)$ cover the critical points $x_{c r}^{(1)}, x_{c r}^{(2)}, "\left(M_{0}, \zeta_{0}, \delta ; x_{0}\right)$-regularly" and " $\left(M_{0}, \zeta_{0}, \delta ; h\left(x_{0}\right)\right)$-regularly", respectively.

Lemma 3.4. Suppose the circle homeomorphisms $f_{1}$ and $f_{2}$ satisfy the conditions of Theorem 1.4. Then there exists natural number $k_{0}$ such that for intervals $\left[z_{s}, z_{s+1}\right], s=1,2,3$ satisfying conditions (1)-(3) of Lemma 3.3, and for sufficiently large $n$ the following inequality holds

$$
\left|\frac{\operatorname{Dist}\left(z_{1}, z_{2}, z_{3}, z_{4} ; f_{1}^{q_{n}}\right)}{\operatorname{Dist}\left(h\left(z_{1}\right), h\left(z_{2}\right), h\left(z_{3}\right), h\left(z_{4}\right) ; f_{2}^{q_{n}}\right)}-1\right| \geqslant R_{2}>0,
$$

where the constant $R_{2}$ depends only on $f_{1}$ and $f_{2}$.

Proof of Theorem 1.4. Let $f_{1}$ and $f_{2}$ be circle homeomorphisms satisfying the conditions of Theorem 1.4. The lift $H(x)$ of the conjugating map $h(x)$ is a continuous and monotone increasing function on $R^{1}$. Hence $H(x)$ has a finite derivative $H^{\prime}(x)$ for almost all $x$ with respect to Lebesgue measure. We claim that $h^{\prime}(x)=0$ at all points $x$ where the finite derivative exists. Suppose $h^{\prime}\left(x_{0}\right)>0$ for some point $x_{0} \in S^{1}$. Fix $\varepsilon>0$. We take a triple of intervals $\left[z_{s}, z_{s+1}\right] \subset$ $\left(x_{0}-\delta, x_{0}+\delta\right), s=1,2,3$, satisfying the conditions of Lemma 3.4.

Using the assertion of Lemma 3.1 we obtain

$$
\begin{gathered}
\left|\operatorname{Dist}\left(z_{1}, z_{2}, z_{3}, z_{4} ; h\right)-1\right| \leqslant C_{3} \varepsilon \\
\left|\operatorname{Dist}\left(f_{1}^{q_{n}}\left(z_{1}\right), f_{1}^{q_{n}}\left(z_{2}\right), f_{1}^{q_{n}}\left(z_{3}\right), f_{1}^{q_{n}}\left(z_{4}\right) ; h\right)-1\right| \leqslant C_{3} \varepsilon .
\end{gathered}
$$

Hence

$$
\left|\frac{\operatorname{Dist}\left(z_{1}, z_{2}, z_{3}, z_{4} ; h\right)}{\operatorname{Dist}\left(f_{1}^{q_{n}}\left(z_{1}\right), f_{1}^{q_{n}}\left(z_{2}\right), f_{1}^{q_{n}}\left(z_{3}\right), f_{1}^{q_{n}}\left(z_{4}\right) ; h\right)}-1\right| \leqslant C_{4} \varepsilon,
$$

where the constant $C_{4}>0$ does not depend on $\varepsilon$ and $n$. 
Since $h$ is conjugating $f_{1}$ and $f_{2}$ we can readily see that

$$
\begin{aligned}
& C r\left(h\left(f_{1}^{q_{n}}\left(z_{1}\right)\right), h\left(f_{1}^{q_{n}}\left(z_{2}\right)\right), h\left(f_{1}^{q_{n}}\left(z_{3}\right)\right), h\left(f_{1}^{q_{n}}\left(z_{4}\right)\right)\right)= \\
& =C r\left(f_{2}^{q_{n}}\left(h\left(z_{1}\right)\right), f_{2}^{q_{n}}\left(h\left(z_{2}\right)\right), f_{2}^{q_{n}}\left(h\left(z_{3}\right)\right), f_{2}^{q_{n}}\left(h\left(z_{4}\right)\right)\right) .
\end{aligned}
$$

Hence we obtain

$$
\begin{gathered}
\frac{\operatorname{Dist}\left(f_{1}^{q_{n}}\left(z_{1}\right), f_{1}^{q_{n}}\left(z_{2}\right), f_{1}^{q_{n}}\left(z_{3}\right), f_{1}^{q_{n}}\left(z_{4}\right) ; h\right)}{\operatorname{Dist}\left(z_{1}, z_{2}, z_{3}, z_{4} ; h\right)}= \\
=\frac{C r\left(h\left(f_{1}^{q_{n}}\left(z_{1}\right)\right), h\left(f_{1}^{q_{n}}\left(z_{2}\right)\right), h\left(f_{1}^{q_{n}}\left(z_{3}\right)\right), h\left(f_{1}^{q_{n}}\left(z_{4}\right)\right)\right)}{\operatorname{Cr}\left(f_{1}^{q_{n}}\left(z_{1}\right), f_{1}^{q_{n}}\left(z_{2}\right), f_{1}^{q_{n}}\left(z_{3}\right), f_{1}^{q_{n}}\left(z_{4}\right)\right)} \times \\
\times \frac{C r\left(z_{1}, z_{2}, z_{3}, z_{4}\right)}{\operatorname{Cr}\left(h\left(z_{1}\right), h\left(z_{2}\right), h\left(z_{3}\right), h\left(z_{4}\right)\right)}=\frac{C r\left(f_{2}^{q_{n}}\left(h\left(z_{1}\right)\right), f_{2}^{q_{n}}\left(h\left(z_{2}\right)\right), f_{2}^{q_{n}}\left(h\left(z_{3}\right)\right), f_{2}^{q_{n}}\left(h\left(z_{4}\right)\right)\right)}{\operatorname{Cr}\left(h\left(z_{1}\right), h\left(z_{2}\right), h\left(z_{3}\right), h\left(z_{4}\right)\right)}: \\
: \frac{\operatorname{Cr}\left(f_{1}^{q_{n}}\left(z_{1}\right), f_{1}^{q_{n}}\left(z_{2}\right), f_{1}^{q_{n}}\left(z_{3}\right), f_{1}^{q_{n}}\left(z_{4}\right)\right)}{C r\left(z_{1}, z_{2}, z_{3}, z_{4}\right)}=\frac{\operatorname{Dist}\left(h\left(z_{1}\right), h\left(z_{2}\right), h\left(z_{3}\right), h\left(z_{4}\right) ; f_{2}^{q_{n}}\right)}{\operatorname{Dist}\left(z_{1}, z_{2}, z_{3}, z_{4} ; f_{1}^{q_{n}}\right)} .
\end{gathered}
$$

This, together with (3.6) obviously implies that

$$
\left|\frac{\operatorname{Dist}\left(z_{1}, z_{2}, z_{3}, z_{4} ; f_{1}^{q_{n}}\right)}{\operatorname{Dist}\left(h\left(z_{1}\right), h\left(z_{2}\right), h\left(z_{3}\right), h\left(z_{4}\right) ; f_{2}^{q_{n}}\right)}-1\right| \leqslant C_{5} \varepsilon,
$$

where the constant $C_{5}>0$ does not depend on $\varepsilon$ and $n$. This contradicts equation (3.3). Therefore Theorem 1.4 is completely proved.

\section{The proofs of Lemmas 3.2-3.4}

Proof of Lemma 3.2. Denote

$$
\psi_{1}\left(\xi_{f_{1}}(l)\right)=\frac{1}{1-\xi_{f_{1}}(l)+\cdots+\xi_{f_{1}}^{2 m_{1}}(l)},
$$

and

$$
\psi_{2}\left(\eta_{f_{1}}(l)\right)=\frac{e_{2 m_{1}} \eta_{f_{1}}^{2 m_{1}}(l)+e_{2 m_{1}-1} \eta_{f_{1}}^{2 m_{1}-1}(l)+\cdots+1}{\eta_{f_{1}}^{2 m_{1}}(l)+C_{2 m_{1}}^{1} \eta_{f_{1}}^{2 m_{1}-1}(l)+\cdots+1} .
$$

It is easy to check that for $\eta_{f_{1}}(l)>0$ the function $\psi_{2}\left(\eta_{f_{1}}(l)\right)$ is monotone increasing and $1<$ $\psi_{2}\left(\eta_{f_{1}}(l)\right)<2 m_{1}+1$. Obviously

$$
\lim _{\xi_{f_{1}}(l) \rightarrow 0} \psi_{1}\left(\xi_{f_{1}}(l)\right)=1, \quad \lim _{\eta_{f_{1}}(l) \rightarrow \infty} \psi_{2}\left(\eta_{f_{1}}(l)\right)=2 m_{1}+1
$$

Taking these remarks into account and using the explicit form of the functions $\psi_{1}\left(\xi_{f_{1}}(l)\right)$ and $\psi_{2}\left(\eta_{f_{1}}(l)\right)$ we can now estimate $\left|\psi_{1} \cdot \psi_{2}-\left(2 m_{1}+1\right)\right|$. Firstly, we estimate $\psi_{2}$ for large value of $\eta_{f_{1}}(l)$. Using the explicit form of the function $\psi_{2}\left(\eta_{f_{1}}(l)\right)$, we see that the inequality

$$
\left|\psi_{2}-\left(2 m_{1}+1\right)\right|=O\left(\frac{1}{\eta_{f_{1}}(l)}\right) \leqslant R_{3}\left(\frac{1}{\eta_{f_{1}}(l)}\right),
$$

where the constant $R_{3}>0$ depends only on $f_{1}$. If we choose $\eta_{f_{1}}(l)$ satisfying the inequality $R_{2}\left(\frac{1}{\eta_{f_{1}}(l)}\right)<\frac{L}{32}$, then

$$
\left|\psi_{2}\left(\eta_{f_{1}}(l)\right)-\left(2 m_{1}+1\right)\right|<\frac{L}{32}
$$


for $\eta_{f_{1}}(l)>\frac{32 R_{3}}{L}$.

We next estimate $\left|\psi_{1}-1\right|$ for small value of $\xi_{f_{1}}(l)$. Using the explicit form of the function $\psi_{1}\left(\xi_{f_{1}}(l)\right)$, we see that $\left|\psi_{1}\left(\xi_{f_{1}}(l)\right)-1\right|=O\left(\xi_{f_{1}}(l)\right) \leqslant R_{4} \xi_{f_{1}}(l)$. It follows from this together with (4.1) that $\left|\psi_{1} \cdot \psi_{2}-\left(2 m_{1}+1\right)\right| \leqslant\left|\psi_{2}-\left(2 m_{1}+1\right)\right|+\left|\psi_{2}\right| \cdot\left|\psi_{1}-1\right| \leqslant \frac{L}{32}+\left(2 m_{1}+1\right) R_{4} \xi_{f_{1}}(l)$. If we take

$$
\zeta_{1}:=\min \left\{\frac{L}{32\left(2 m_{1}+1\right) R_{5}}, 1\right\}, \quad M_{1}:=\max \left\{\frac{32 R_{5}}{L}, 1\right\},
$$

where $R_{5}=\max \left\{R_{3}, R_{4}\right\}$, then for all $\xi_{f_{1}}(l)<\zeta_{1}$ and $\eta_{f_{1}}(l)>M_{1}$ the following inequality holds

$$
\left|\psi_{1} \cdot \psi_{2}-\left(2 m_{1}+1\right)\right| \leqslant \frac{L}{16} .
$$

Similarly it can be shown that with

$$
\zeta_{2}:=\min \left\{\frac{L}{32\left(2 m_{2}+1\right) R_{6}}, 1\right\}, \quad M_{2}:=\max \left\{\frac{32 R_{6}}{L}, 1\right\},
$$

and $\xi_{f_{2}}(l)<\zeta_{2}$ and $\eta_{f_{2}}(l)>M_{2}$, the second assertion of Lemma 3.2 holds. In (4.2) the constants $R_{6}>0$ depends only on $f_{2}$. Finally, if we set $\zeta_{0}:=\min \left\{\zeta_{1}, \zeta_{2}\right\}$ and $M_{0}:=\max \left\{M_{1}, M_{2}\right\}$, then Lemma 3.2 holds for $\xi_{f_{1}}(l), \xi_{f_{2}}(l) \in\left[0, \zeta_{0}\right)$ and $\eta_{f_{1}}(l), \eta_{f_{2}}(l) \geqslant M_{0}$. Lemma 3.2 is proved.

Proof of Lemma 3.3. Firstly, we prove the third assertion of the lemma. By the construction of the points $z_{i}, i=1,2,3,4$, it implies that the intervals $\left[z_{s}, z_{s+1}\right]$ and $\left[h\left(z_{s}\right), h\left(z_{s+1}\right)\right], s=1,2,3$ satisfy the 1) and 2) conditions of definition of "regularly" covering. We consider dynamical partition $\xi_{n}\left(x_{c r}^{(1)}\right)$. According to Lemma 2.2 the intervals $\Delta_{0}^{(n)}\left(x_{c r}^{(1)}\right)$ and $\Delta_{0}^{(n-1)}\left(x_{c r}^{(1)}\right)$ are $K$ comparable, i.e. there exist constant $K>1$ such that $K^{-1} \ell\left(\Delta_{0}^{(n-1)}\left(x_{c r}^{(1)}\right)\right) \leqslant \ell\left(\Delta_{0}^{(n)}\left(x_{c r}^{(1)}\right)\right) \leqslant$ $K \ell\left(\Delta_{0}^{(n-1)}\left(x_{c r}^{(1)}\right)\right)$. Thus it follows that there exists $k_{1}^{(1)} \in N$ such that the following inequality holds

$$
\frac{\ell\left(\left[x_{c r}^{(1)}, f_{1}^{q}{ }^{n+k_{0}+k_{1}^{(1)}}\left(x_{c r}^{(1)}\right)\right]\right)}{\ell\left(\left[f_{1}^{q_{n+k_{0}}}\left(x_{c r}^{(1)}\right), x_{c r}^{(1)}\right]\right)}<\zeta_{0} .
$$

Indeed, it is clear that

$$
\frac{\ell\left(\Delta_{0}^{\left(q_{\left.n+k_{0}+3\right)}\right)}\left(x_{c r}^{(1)}\right)\right)}{\ell\left(\Delta_{0}^{\left(q_{\left.n+k_{0}+1\right)}\right)}\left(x_{c r}^{(1)}\right)\right)}=\frac{1}{\frac{\ell\left(\Delta_{0}^{\left(q_{n+k}+1\right)}\left(x_{c r}^{(1)}\right)\right)}{\ell\left(\Delta_{0}^{\left(q_{n+k}+k_{0}+3\right)}\left(x_{c r}^{(1)}\right)\right)}} \leqslant \frac{1}{1+\frac{1}{K}}=\frac{K}{K+1} .
$$

Hence $\ell\left(\Delta_{0}^{\left(q_{\left.n+k_{0}+3\right)}\right)}\left(x_{c r}^{(1)}\right)\right) \leqslant \frac{K}{K+1} \ell\left(\Delta_{0}^{\left(q_{n+k_{0}+1}\right)}\left(x_{c r}^{(1)}\right)\right)$. Using the last inequality we obtain that for any $k$

$$
\ell\left(\Delta_{0}^{\left(q_{\left.n+k_{0}+k\right)}\right.}\left(x_{c r}^{(1)}\right)\right) \leqslant\left(\frac{K}{K+1}\right)^{k} \ell\left(\Delta_{0}^{\left(q_{\left.n+k_{0}+1\right)}\right.}\left(x_{c r}^{(1)}\right)\right) .
$$

Since $\Delta_{0}^{\left(q_{n+k_{0}+1}\right)}\left(x_{c r}^{(1)}\right)$ and $\Delta_{0}^{\left(q_{n+k_{0}}\right)}\left(x_{c r}^{(1)}\right)$ are $K$-comparable, there exists a $k_{1}^{(1)} \in N$ such that the inequality (4.3) is true. Similarly, we can show that there exists a $k_{2}^{(1)} \in N$ such that the following inequality holds

$$
\frac{\ell\left(\left[x_{c r}^{(1)}, f_{1}^{q_{n+k_{0}+k_{1}}}\left(x_{c r}^{(1)}\right)\right]\right)}{\ell\left(\left[f_{1}^{q_{n+k_{0}+k_{1}}}\left(x_{c r}^{(1)}\right), f_{1}^{q_{n+k_{0}+k_{1}}^{(1)}+q_{n+k_{2}}^{(1)}}\left(x_{c r}^{(1)}\right)\right]\right)}>M_{0} .
$$


Similarly, it can be shown that with natural numbers $k_{1}^{(2)}$ and $k_{1}^{(2)}$ the inequalities

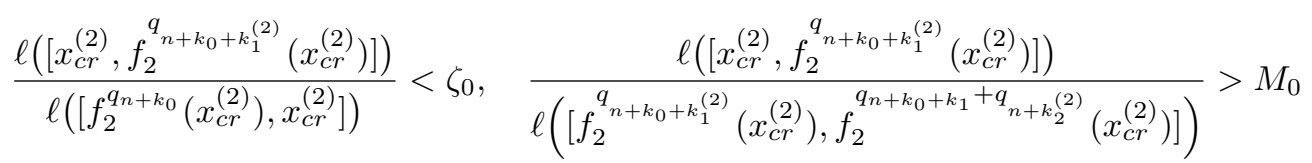

hold. If we take $k_{1}=\max \left\{k_{1}^{(1)}, k_{1}^{(2)}\right\}$ and $k_{2}=\max \left\{k_{2}^{(1)}, k_{2}^{(2)}\right\}$ then the third assertion of Lemma 3.3 holds for $k_{1}$ and $k_{2}$. By the definition of the points $z_{i}, i=1,2,3$ it implies the first assertion of the lemma.

Let $\xi_{n}\left(\bar{x}_{c r}^{(1)}\right)$ be a dynamical partition of the point $\bar{x}_{c r}^{(1)}$. According to Lemma 2.2 the intervals $\Delta_{0}^{(n)}\left(\bar{x}_{c r}^{(1)}\right)$ and $\Delta_{0}^{(n-1)}\left(\bar{x}_{c r}^{(1)}\right)$ are $K$-comparable. Hence, it implies that the intervals $\left[z_{s}, z_{s+1}\right], s=1,2,3$ are pairwise $K^{k_{1}+k_{2}}$ comparable. It is easy to see that the intervals $\left[f_{1}^{q_{n}}\left(z_{s}\right), f_{1}^{q_{n}}\left(z_{s+1}\right)\right], s=1,2,3$ are pairwise $K^{k_{1}+k_{2}}$-comparable. Obviously,

$$
\frac{1}{K^{k_{0}+1}} \leqslant \frac{\ell\left(\Delta_{0}^{(n-1)}\left(\bar{x}_{c r}^{(1)}\right)\right)}{\ell\left(\left[z_{1}, z_{2}\right]\right)} \leqslant K^{k_{0}+1}, \quad \frac{1}{K^{k_{0}+1}} \leqslant \frac{\ell\left(\Delta_{0}^{(n-1)}\left(\bar{x}_{c r}^{(1)}\right)\right)}{\ell\left(\left[f_{1}^{q_{n}}\left(z_{1}\right), f_{1}^{q_{n}}\left(z_{2}\right)\right]\right)} \leqslant K^{k_{0}+1} .
$$

Since the intervals $\Delta_{0}^{(n-1)}\left(\bar{x}_{c r}^{(1)}\right)$ and $\Delta_{0}^{(n-1)}\left(f_{1}^{-q_{n-1}}\left(\bar{x}_{c r}^{(1)}\right)\right)$ are $K$-comparable and $x_{0} \in \Delta_{0}^{(n-1)}\left(f_{1}^{-q_{n-1}}\left(\bar{x}_{c r}^{(1)}\right)\right) \cup \Delta_{0}^{(n-1)}\left(\bar{x}_{c r}^{(1)}\right)$ we get

$$
\max _{1 \leqslant i \leqslant 4}\left\{\ell\left(\left[f^{q_{n}}\left(z_{i}\right), x_{0}\right]\right), \ell\left(\left[z_{i}, x_{0}\right]\right)\right\} \leqslant(K+1) K^{k_{0}+1} \ell\left(\left[z_{1}, z_{2}\right]\right) .
$$

If we take $R_{1}=(K+1) K^{k_{0}+k_{1}+k_{2}}$, then we obtain the proof of the second assertion of Lemma 3.3 with constant $R_{1}$. Lemma 3.3 is proved.

Proof of Lemma 3.4. Suppose, the triples of intervals $\left(\left[z_{s}, z_{s+1}\right], s=1,2,3\right)$ and $\left(\left[h\left(z_{s}\right), h\left(z_{s+1}\right)\right]\right.$, $s=1,2,3)$ satisfy the conditions of Lemma 3.3. We want to compare the distortion $\operatorname{Dist}\left(z_{1}, z_{2}, z_{3}, z_{4} ; f_{1}^{q_{n}}\right)$ and $\operatorname{Dist}\left(h\left(z_{1}\right), h\left(z_{2}\right), h\left(z_{3}\right), h\left(z_{4}\right) ; f_{2}^{q_{n}}\right)$. We estimate only the first distortion, the second one can be estimated analogously. Obviously

We denote

$$
\operatorname{Dist}\left(z_{1}, z_{2}, z_{3}, z_{4} ; f_{1}^{q_{n}}\right)=\prod_{i=0}^{q_{n}-1} \operatorname{Dist}\left(f_{1}^{i}\left(z_{1}\right), f_{1}^{i}\left(z_{2}\right), f_{1}^{i}\left(z_{3}\right), f_{1}^{i}\left(z_{4}\right) ; f_{1}\right) .
$$

$$
\begin{gathered}
J_{r}\left(x_{c r}^{(1)}\right)=\Delta_{0}^{(r)}\left(x_{c r}^{(1)}\right) \cup \Delta_{0}^{(r-1)}\left(x_{c r}^{(1)}\right), \quad A=\left\{i:\left(f_{1}^{i}\left(z_{1}\right), f_{1}^{i}\left(z_{4}\right)\right) \cap J_{r}\left(x_{c r}^{(1)}\right)=\emptyset\right\}, \\
B=\left\{i:\left(f_{1}^{i}\left(z_{1}\right), f_{1}^{i}\left(z_{4}\right)\right) \cap J_{r}\left(x_{c r}^{(1)}\right) \neq \emptyset\right\} .
\end{gathered}
$$

It is clear that $A \cup B=\left\{0,1, \ldots, q_{n}\right\}$.

Next we rewrite $\operatorname{Dist}\left(z_{1}, z_{2}, z_{3}, z_{4} ; f_{1}^{q_{n}}\right)$ in the form

$$
\begin{gathered}
\operatorname{Dist}\left(z_{1}, z_{2}, z_{3}, z_{4} ; f_{1}^{q_{n}}\right)=\prod_{i \in A} \operatorname{Dist}\left(f_{1}^{i}\left(z_{1}\right), f_{1}^{i}\left(z_{2}\right), f_{1}^{i}\left(z_{3}\right), f_{1}^{i}\left(z_{4}\right) ; f_{1}\right) \times \\
\times \prod_{i \in B} \operatorname{Dist}\left(f_{1}^{i}\left(z_{1}\right), f_{1}^{i}\left(z_{2}\right), f_{1}^{i}\left(z_{3}\right), f_{1}^{i}\left(z_{4}\right) ; f_{1}\right) .
\end{gathered}
$$

We estimate the first factor in (4.4). Using the Lemmas 2.4 we obtain

$$
\begin{gathered}
\left|\prod_{i \in A} \operatorname{Dist}\left(f_{1}^{i}\left(z_{1}\right), f_{1}^{i}\left(z_{2}\right), f_{1}^{i}\left(z_{3}\right), f_{1}^{i}\left(z_{4}\right) ; f_{1}\right)-1\right|=\left|\prod_{i \in A}\left(1+O\left(\ell\left(\left[f_{1}^{i}\left(z_{1}\right), f_{1}^{i}\left(z_{4}\right)\right]\right)\right)^{1+\nu}\right)-1\right|= \\
=\max _{i}\left(\ell\left(\left[f_{1}^{i}\left(z_{1}\right), f_{1}^{i}\left(z_{4}\right)\right]\right)\right)^{\nu} O\left(\sum_{i \in A} \ell\left(\left[f_{1}^{i}\left(z_{1}\right), f_{1}^{i}\left(z_{4}\right)\right]\right)\right)=O\left(\lambda_{f_{1}}^{n \nu}\right), \\
-297-
\end{gathered}
$$


where $\nu>0$ and $0<\lambda_{f_{1}}<1$. We fix $\varepsilon>0$. There exists $N_{0}=N_{0}(\varepsilon) \geqslant 1$ such that for any $n \geqslant N_{0}$ the estimate

$$
\left|\prod_{i \in A} \operatorname{Dist}\left(f_{1}^{i}\left(z_{1}\right), f_{1}^{i}\left(z_{2}\right), f_{1}^{i}\left(z_{3}\right), f_{1}^{i}\left(z_{4}\right) ; f_{1}\right)-1\right|<C_{6} \varepsilon
$$

holds. We now estimate the second factor in (4.4). We rewrite the second factor in the following form

$$
\begin{gathered}
\prod_{i \in B} \operatorname{Dist}\left(f_{1}^{i}\left(z_{1}\right), f_{1}^{i}\left(z_{2}\right), f_{1}^{i}\left(z_{3}\right), f_{1}^{i}\left(z_{4}\right) ; f_{1}\right)= \\
=\prod_{i \in B, i \neq l} \operatorname{Dist}\left(f_{1}^{i}\left(z_{1}\right), f_{1}^{i}\left(z_{2}\right), f_{1}^{i}\left(z_{3}\right), f_{1}^{i}\left(z_{4}\right) ; f_{1}\right) \times \operatorname{Dist}\left(f_{1}^{l}\left(z_{1}\right), f_{1}^{l}\left(z_{2}\right), f_{1}^{l}\left(z_{3}\right), f_{1}^{l}\left(z_{4}\right) ; f_{1}\right) .
\end{gathered}
$$

By applying Lemmas 2.5 and 3.2 we obtain

$$
\left|\operatorname{Dist}\left(f_{1}^{l}\left(z_{1}\right), f_{1}^{l}\left(z_{2}\right), f_{1}^{l}\left(z_{3}\right), f_{1}^{l}\left(z_{4}\right) ; f_{1}\right)-\left(2 m_{1}+1\right)\right|<\frac{L}{8} .
$$

Using Lemma 2.6 for the first factor in (4.6), we get

$$
\begin{gathered}
\left|\prod_{i \in B, i \neq l} \operatorname{Dist}\left(f_{1}^{i}\left(z_{1}\right), f_{1}^{i}\left(z_{2}\right), f_{1}^{i}\left(z_{3}\right), f_{1}^{i}\left(z_{4}\right) ; f_{1}\right)-1\right|=\left|\prod_{i \in B, i \neq l}\left(1+O\left(\frac{\ell\left(\left[f_{1}^{i}\left(z_{1}\right), f_{1}^{i}\left(z_{4}\right)\right]\right)}{d_{i}}\right)^{2}\right)-1\right|= \\
=\left|\exp \left\{\sum_{i \in B, i \neq l} \log \left(1+O\left(\frac{\ell\left(\left[f_{1}^{i}\left(z_{1}\right), f_{1}^{i}\left(z_{4}\right)\right]\right)}{d_{i}}\right)^{2}\right)\right\}-1\right| \leqslant \operatorname{const} \sum_{i \in B, i \neq l}\left(\frac{\ell\left(\left[f_{1}^{i}\left(z_{1}\right), f_{1}^{i}\left(z_{4}\right)\right]\right)}{d_{i}}\right)^{2}= \\
=\operatorname{const} \sum_{q=0}^{n-r}\left(\frac{\ell\left(\left[f_{1}^{i}\left(z_{1}\right), f_{1}^{i}\left(z_{4}\right)\right]\right)}{d_{i}}\right)^{2} . \\
\sum_{i:\left[f_{1}^{i}\left(z_{1}\right), f_{1}^{i}\left(z_{4}\right)\right] \subset\left(J_{n-q}\left(x_{c r}^{(1)}\right) \backslash J_{n-q+1}\left(x_{c r}^{(1)}\right)\right), i \neq l}
\end{gathered}
$$

Obviously,

$$
\sum_{i:\left[f_{1}^{i}\left(z_{1}\right), f_{1}^{i}\left(z_{4}\right)\right] \subset\left(J_{n-q}\left(x_{c r}^{(1)}\right) \backslash J_{n-q+1}\left(x_{c r}^{(1)}\right)\right), i \neq l}\left(\frac{\ell\left(\left[f_{1}^{i}\left(z_{1}\right), f_{1}^{i}\left(z_{4}\right)\right]\right)}{d_{i}}\right)=\text { const }
$$

and it follows from Lemma 2.3 that $\frac{\ell\left(\left[f_{1}^{i}\left(z_{1}\right), f_{1}^{i}\left(z_{4}\right)\right]\right)}{d_{i}} \leqslant$ const $\lambda_{f_{1}}^{k_{0}+1+q}$. Consequently

$$
\left|\prod_{i \in B, i \neq l} \operatorname{Dist}\left(f_{1}^{i}\left(z_{1}\right), f_{1}^{i}\left(z_{2}\right), f_{1}^{i}\left(z_{3}\right), f_{1}^{i}\left(z_{4}\right) ; f_{1}\right)-1\right| \leqslant C_{7} \lambda_{f_{1}}^{k_{0}},
$$

where $C_{7}>0$ depends only on $f_{1}$.

Similarly one can show that for the triple of intervals $\left(\left[h\left(z_{s}\right), h\left(z_{s+1}\right)\right], s=1,2,3\right)$ the following inequality

$$
\left|\prod_{i \in B, i \neq l} \operatorname{Dist}\left(f_{2}^{i}\left(h\left(z_{1}\right)\right), f_{2}^{i}\left(h\left(z_{2}\right)\right), f_{2}^{i}\left(h\left(z_{3}\right)\right), f_{2}^{i}\left(h\left(z_{4}\right)\right) ; f_{2}\right)-1\right| \leqslant C_{8} \lambda_{f_{2}}^{k_{0}},
$$

where $C_{8}>0$ depends only on $f_{2}$ and $0 \leqslant \lambda_{f_{2}} \leqslant 1$ is defined in Lemma 2.3.

If we choose

$$
k_{0}=\max \left\{\left[\log _{\lambda_{f_{1}}} \frac{L}{\left(16 m_{1}+8+L\right) C_{7}}\right]+1,\left[\log _{\lambda_{f_{2}}} \frac{L}{\left(16 m_{2}+8+L\right) C_{8}}\right]+1\right\},
$$


where constants $0 \leqslant \lambda_{f_{1}}, \lambda_{f_{2}} \leqslant 1$ are defined in Lemma 2.3, then from the relations (4.4)-(4.8) it implies that for sufficiently large $n$

$$
\left|\operatorname{Dist}\left(z_{1}, z_{2}, z_{3}, z_{4} ; f_{1}^{q_{n}}\right)-\left(2 m_{1}+1\right)\right|<\frac{L}{4} .
$$

Similarly

$$
\left|\operatorname{Dist}\left(h\left(z_{1}\right), h\left(z_{2}\right), h\left(z_{3}\right), h\left(z_{4}\right) ; f_{2}^{q_{n}}\right)-\left(2 m_{2}+1\right)\right|<\frac{L}{4} .
$$

The inequalities (4.10) and (4.11) implies

$$
\frac{\operatorname{Dist}\left(z_{1}, z_{2}, z_{3}, z_{4} ; f_{1}^{q_{n}}\right)}{\operatorname{Dist}\left(h\left(z_{1}\right), h\left(z_{2}\right), h\left(z_{3}\right), h\left(z_{4}\right) ; f_{2}^{q_{n}}\right)}-1 \geqslant \frac{8\left(m_{1}-m_{2}\right)-2 L}{8 m_{2}+L+4}>0,
$$

if $m_{1}>m_{2}$, and

$$
\frac{\operatorname{Dist}\left(z_{1}, z_{2}, z_{3}, z_{4} ; f_{1}^{q_{n}}\right)}{\operatorname{Dist}\left(h\left(z_{1}\right), h\left(z_{2}\right), h\left(z_{3}\right), h\left(z_{4}\right) ; f_{2}^{q_{n}}\right)}-1 \leqslant \frac{8\left(m_{1}-m_{2}\right)+2 L}{8 m_{2}-L+4}<0,
$$

if $m_{1}<m_{2}$. If we set

$$
R_{2}:=\min \left\{\frac{\left|8\left(m_{1}-m_{2}\right)-2 L\right|}{8 m_{2}-L+4}, \frac{\left|8\left(m_{1}-m_{2}\right)+2 L\right|}{8 m_{2}+L+4}\right\},
$$

then it follows from (4.12)-(4.14) that the assertion of the lemma holds.

The author would like to thank Professors A. A. Dzhalilov, K. M. Khanin and A. Davydov for useful discussion.

\section{References}

[1] V.I.Arnol'd, Small denominators: I. Mappings from the circle onto itself, Izv. Akad. Nauk SSSR, Ser. Mat., 25(1961), 21-86 (in Russian).

[2] A.Avila, On rigidity of critical circle maps, Bull. Math. Soc., 44(2013), no. 4, 611-619. DOI: $10.1007 / \mathrm{s} 00574-013-0027-5$

[3] I.P.Cornfeld, S.V.Fomin, Ya.G.Sinai, Ergodic Theory, Springer Verlag, Berlin, 1982.

[4] A.Denjoy, Sur les courbes définies par les équations différentielles à la surface du tore, $J$. Math. Pures Appl., 11(1932), 333-375.

[5] A.A.Dzhalilov, K.M.Khanin, On invariant measure for homeomorphisms of a circle with a point of break, Funct. Anal. Appl., 32(1998), no. 3, 153-161.

[6] E. de Faria, W. de Melo, Rigidity of critical circle mappings I, J. Eur. Math. Soc. (JEMS), 1(1999), no. 4, 339-392.

[7] J.Graczyk, G.Swiatek, Singular measures in circle dynamics, Commun. Math. Phys., 157(1993), 213-230.

[8] P.Guarino, M.Martens, W. de Melo, Rigidity of critical circle maps, Duke Math. J., 167(2018), 2125-2188. DOI: 10.1215/00127094-2018-0017 
[9] M.Herman, Sur la conjugaison différentiable des difféomorphismes du cercle à des rotations, Inst. Hautes Etudes Sci. Publ. Math., 49(1979), 225-234.

[10] K.Khanin, Universal estimates for critical circle mappings, CHAOS, 2(1991), 181-186.

[11] K.Khanin, A.Teplinskii, Robust rigidity for circle diffeomorphisms with singularities, Invent. math., 169(2007), 193-218. DOI: 10.1007/s00222-007-0047-0

[12] K.M.Khanin, Ya.G.Sinai, Smoothness of conjugacies of diffeomorphisms of the circle with rotations, Usp. Mat. Nauk, 44(1989), 57-82.

[13] Y.Katznelson, D.Ornstein, The absolute continuity of the conjugation of certain diffeomorphisms of the circle, Ergod. Theor. Dyn. Syst., 9(1989), 681-690.

[14] D.Khmelev, M.Yampolski, Rigidity problem for analytic critical circle maps, Mos. Math. Journ., 6(2006), no. 2, 317-351.

[15] J.Moser, A rapid convergent iteration method and non-linear differential equations, II. Ann. Scuola Norm. Sup. Pisa, 20(1966), no. 3, 499-535.

[16] G.Swiatek, Rational rotation numbers for maps of the circle, Commun. Math. Phys., 119(1988), no. 1, 109-128.

[17] J.C.Yoccoz, Il n'a a pas de contre-exemple de Denjoy analytique, C. R. Acad. Sci., Paris, Ser.I, Math., 298(1984), no. 7, 141-144.

\title{
О сопряжение между двумя критическими отображениями окружности
}

\author{
Уткир А. Сафаров \\ Туринский политехнический университет \\ Ташкент, Узбекистан \\ Ташкентский государственный экономический университет \\ Ташкент, Узбекистан
}

\begin{abstract}
Аннотация. В статье изучается сопряжение между двумя критическими гомеоморфизмами окружности с иррациональным числом вращения. Пусть $f_{i}, i=1,2$ являются $C^{3}$-гомеоморфизмы окружности с критической точкой $x_{c r}^{(i)}$ порядка $2 m_{i}+1$. Доказано, что если $2 m_{1}+1 \neq 2 m_{2}+1$, то сопряжение между $f_{1}$ и $f_{2}-$ сингулярная функция.

Ключевые слова: гомеоморфизм окружности, критическая точка, сопрягащий гомеоморфизм, число вращения, сингулярная функция.
\end{abstract}

